\title{
Detection and Alleviation of Driving Fatigue Based on EMG and EMS/EEG Using Wearable Sensor
}

\author{
Hong Wang*, Zuoqiu Qi, Rongrong Fu, Fuwang Wang, Qingwen Yu and Chong Liu \\ School of Mechanical Engineering and Automation, Northeastern University, Shenyang, China \\ *hongwang@mail.neu.edu.cn
}

\begin{abstract}
We present a new technique to detect and relieve drivers' fatigue by using electromyography (EMG) and electrical muscle stimulation (EMS) respectively, while drivers are driving without any interruption. Driving fatigue was detected by EMG from the biceps femoris of drivers' legs through a noncontact acquisition system. The peak factors $(F c)$ of the EMG were used as the feature of driving fatigue state. When the threshold of $F c$ is met or exceeded, the abductor pollicis muscle and thumb flexor muscle of drivers' hands can be stimulated by EMS without affecting the normal driving. The experiment results show that the brain fatigue of drivers can be effectively alleviated by this EMS while driving.
\end{abstract}

\section{Keywords}

EMG, Electrical muscle stimulation, EEG, Driving fatigue, Highway

\section{INTRODUCTION}

With the development of motor industry and transportation, traffic safety is becoming a serious problem. Traffic accidents have caused enormous damage to family and society. According to relative report, driver fatigue is the main cause of traffic accidents [1]. Therefore, it is very important to detect driving fatigue and then alleviate this fatigue efficiently.

A driver's physiological state can be measured in various ways. The sensor such as the electromyography (EMG) is the popular method for evaluating the muscle tension, and is much less expensive and more portable [2]. The electrical muscle stimulation can enhance the muscle and brain activity [3-4]. EEG is also an electrical change recorded from the brain in relation to an event that occurs either in the external world or within the brain itself. EEG could describe the brain fatigue state [5-9]. Could the EMS alleviate the driving fatigue? Here, we have studied how to detect and relieve driver fatigue by using EMG and EMS/EEG respectively, while drivers are driving without any interruption. The relationship between the EMG and EMS/EEG was reported.

\section{EXPERIMENTS}

The whole system is composed of two parts, including the noncontact detection of driving fatigue based on EMG and the driving fatigue alleviation by EMS. In this study there were two types of the experiments.

One type of the experiment was the bus driving on the highway (about $252 \mathrm{~km})$ from Shenyang $\left(41.93^{\circ}\right.$ north latitude, $123.43^{\circ}$ east longitude) to Dandong (40.04 north latitude, $124.35^{\circ}$ east longitude), China (Fig.1). Twenty healthy male subjects (age: 24 $\pm 2.76)$ participated in the experiments. The experiment time involved $2-2.5 \mathrm{~h}$ per subject. For the noncontact detection of driving fatigue, the surface EMG recording electrodes (two pieces of conductive knit fabric of silver-plated nylon with the size of 12 $\mathrm{cm} \times 22 \mathrm{~cm}$ and a surface resistivity of $<1 \Omega / \mathrm{sq}$ ) were placed on the top surface of car seat cushion, while the reference electrode was located on the left ankle bone. The physiological signals were then acquired from the biceps femoris muscle of each leg without direct contact while the subjects were driving without any interruption. In the driving section, every $10 \mathrm{~min}$, 30-s length of data were extracted. Other two 30-s length of data were also extracted from post-driving part and post-rest sitting part, respectively. Fast independent component analysis (Fast ICA) and digital filter were utilized to process the original signals. The peak factor of EMG can be defined as follow:

$$
F c=A / x
$$

where $A$ is the amplitude of EMG and $x$ is the square root of EMG. $F c$ was selected as the characteristic feature to detect fatigue of drivers based on the statistical analysis results given by Kolmogorov-Smirnov $Z$ test.

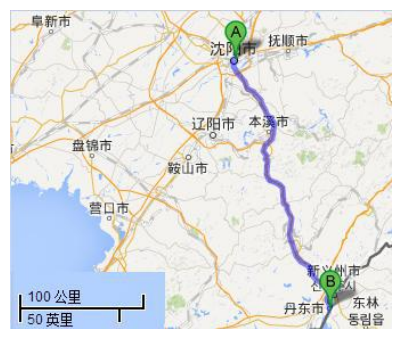

(a)

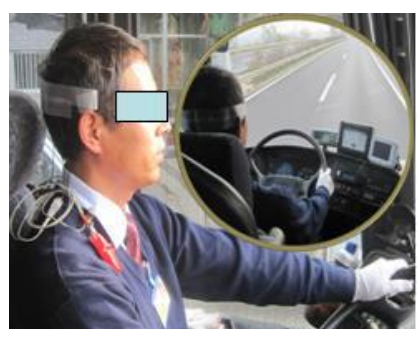

(b)

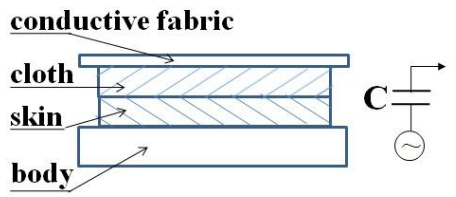

(c)

Fig.1 Bus driving on the highway (about $252 \mathrm{~km}$ ) from Shenyang ( $41.93^{\circ}$ north latitude, $123.43^{\circ}$ east longitude) to Dandong ( $40.04^{\circ}$ north latitude, $124.35^{\circ}$ east longitude), China; (a) the driving rout, 
(b) the bus driver, (c) schematic diagram of the noncontact acquisition system for EMG.

The other type of the experiment was the simulation driving on the driving simulation (Fig.2). Fifty healthy subjects (twenty males and thirty females, age: $22 \pm 2.10$ ) were included in the study. They were recruited within the university and free of medication at the time of the recording session, as well as had no history of neurological diseases.

For the driving fatigue alleviation by EMS, the two conductive knit fabrics were fixed on the steering wheel as stimulation electrodes (Fig.2). When the threshold of $F c$ was met or exceeded, the abductor pollicis muscle and thumb flexor muscle in drivers' hands can be stimulated by EMS without affecting the normal driving. Bipolar square wave pulses were as the stimulation current. The pulse amplitudes within the range from 1 to $3 \mathrm{~mA}$ were chosen according to the comfort of subjects, and the pulse (0.2 ms width) frequency was set as $1 \mathrm{~Hz}$. The effect of the driving fatigue alleviation was evaluated by the features of electroencephalogram (EEG) evoked by EMS. Meanwhile, a mobile phone can also send out a sound alarm about the driving fatigue. In this work, after a one-hour drive, the abductor pollicis muscle and thumb flexor muscle in the experiment group began to be stimulated by EMS without affecting the normal driving. The control group chose normal driving during the experiment. $\theta$ subband $(4 \sim 8 \mathrm{~Hz})$ and $\beta$ subband $(12 \sim 32 \mathrm{~Hz})$ of EEG from the subjects were selected as the evaluation criterion for judging the effect of the driving fatigue alleviation by using EMS. In this experiment, every $12 \mathrm{~min}$, 3-min length of EEG data were extracted.

\section{RESULTS}

The signals recorded by two channels from biceps femoris muscles of the subject while driving are shown in Fig. 3 (A and B). The signals included EMG, electrocardiograph (ECG) and noises. Fast ICA was used to extract EMG (Fig. 3D) from the raw data. The peak factors $(F c)$ from the EMG show upward trend as the driving time increases ( $p<0.05$, Fig. 4$)$.

The effects of driving fatigue alleviation using EMS are shown in both Fig. 5 and Fig. 6 . In control group, the relative power spectra of $\theta$ subband in EEG present general upward trend while increasing the driving time (Fig. 5). In EMS group, during the EMS section, the relative power spectra of $\theta$ subband in EEG show a downward trend until the alert driving state at the beginning of the experiment (Fig. 5). There is a significant difference of the relative power spectra between EMS group and control group during the EMS section ( $p<0.05$, Fig. 5). The relative power spectrum ratio of $\theta / \beta$ is shown in Fig. 6 .

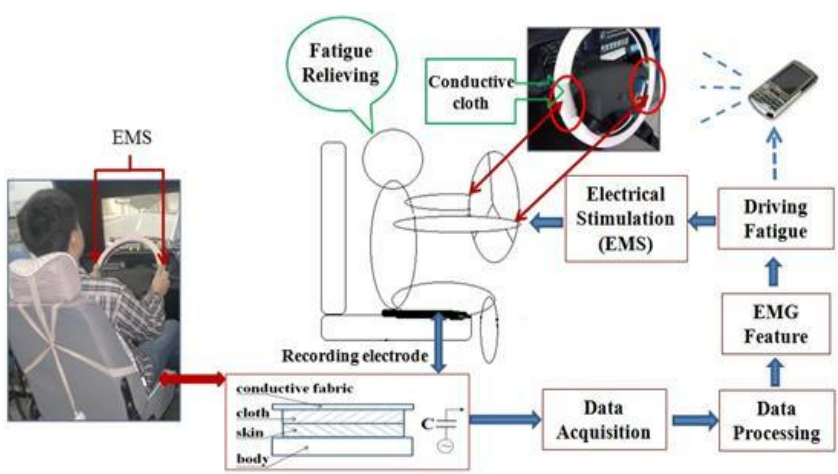

Fig.2 Schematic diagram of the noncontact detection for driving fatigue by EMG and driving fatigue alleviation by EMS.

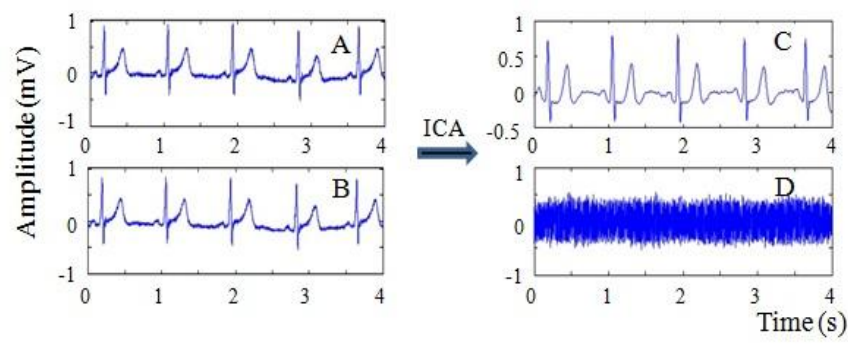

Fig.3 The two-channel raw signals (A, B) from biceps femoris muscles while driving, ECG (C) and EMG (D) separated by Fast ICA from A and B.

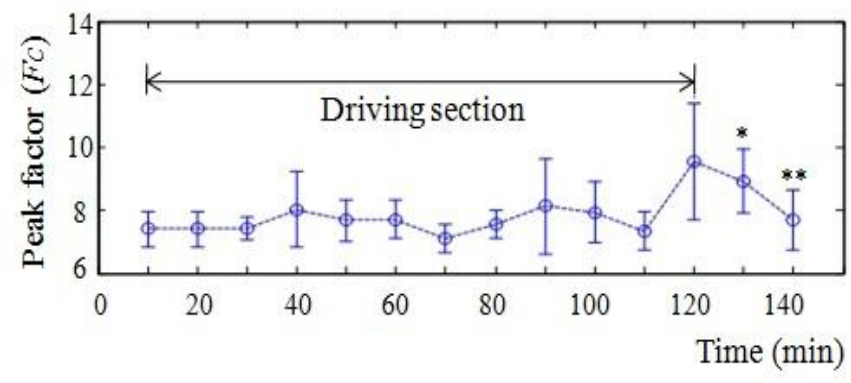

Fig. 4 The peak factor $(F c)$ (mean \pm S.D.) from the EMG. Abscissa axis shows the calculation time of $F c$ (*post-driving section, $* *$ post-rest sitting data).

\section{DISCUSSION}

In this work, the noncontact detection of driving fatigue based on EMG and the driving fatigue alleviation by using EMS are presented. In order to collect physiological signals from subjects while they were driving without any interruption, the system adopted a type of non-contact electrode based on the capacity coupling theory. Recording sensors were placed into the surface of car cushion to collect physiological signals from biceps femoris of each driver, which has made the long-term collection easy to realize. The peak factor $(F c)$ of EMG from the biceps femoris muscle can be selected as the characteristic feature to detect fatigue of drivers. The results show that the method proposed can give well performance in distinguishing the normal state and fatigue state. 
The brain fatigue of drivers can be effectively relieved by the electrical stimulation to the abductor pollicis muscle and thumb flexor muscle. The EMS could evoke less slow waves $(\theta)$ and more fast waves ( $\beta$ ). Theta wave from brain should be produced when human is the shallow sleep state or half awaken consciousness or unconscious activity. Beta wave from brain should be produced when human is consciousness and nervous tension. The inhibition of theta wave from brain could show that the EMS can inhibit the unconscious activity of brain. This means that EMS could lead to cognitive enhancement and may help remission of the driver fatigue. Our experiment results could have guiding significance on the detection and alleviation of driving fatigue.

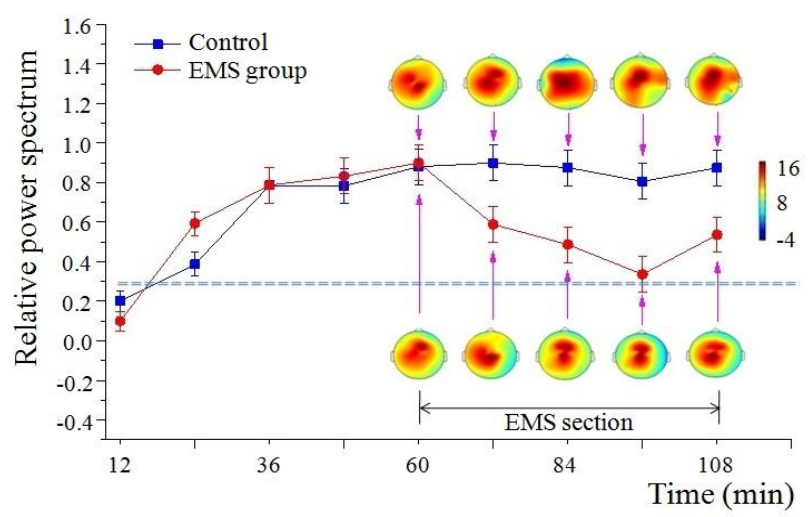

Fig.5 Relative power spectrum (mean \pm S.D.) of $\theta$ subband in EEG as the evaluation of effect of EMS. Abscissa axis shows the recording time of EEG.

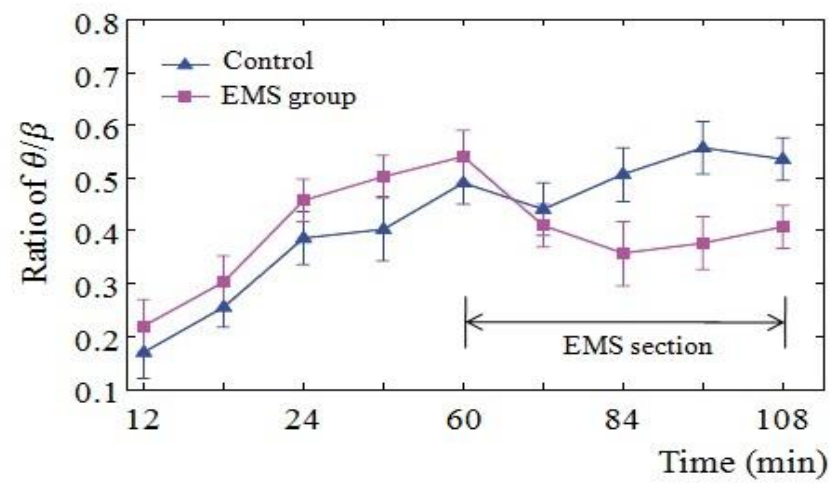

Fig.6 Relative power spectrum ratio $\theta / \beta$ (mean \pm S.D.) as the evaluation of effect of EMS. Abscissa axis shows the recording time of EEG.

\section{ACKNOWLEDGMENTS}

We gratefully acknowledge the financial support of K. C. Wong Education Foundation and the State Key Laboratory of Process Industry Automation of China (PAL-N201304).

\section{REFERENCES}

[1] Gastaldi M, Rossi R and Gecchele G. 2014. Effects of driver task-related fatigue on driving performance. Procedia-Social and Behavioral Sciences. 111 (February 2014): 955-964. DOI= http://dx.doi.org/10.1016/j.sbspro.2014.01.130.

[2] Cronin NJ, Kumpulainen S, Joutjaervi T, Finni $\mathrm{T}$ and Piitulainen H. 2015. Spatial variability of muscle activity during human walking: The effects of different EMG normalization approaches. Neuroscience. 300 (August 2015): 19-28.

$\mathrm{DOI}=$ http://dx.doi.org/10.1016/j.neuroscience.2015.05.003.

[3] Guzman M, Rubin A, Cox P, Landini F and Jackson-Menaldi C.2014. Neuromuscular electrical stimulation of the cricothyroid muscle in patients with suspected superior laryngeal nerve weakness. Journal of Voice. 28 (March 2014): 216-225. DOI= http://dx.doi.org/10.1016/j.jvoice.2013.09.003.

[4] Kim YHB, Lonergan SM, Grubbs JK, Cruzen SM, Fritchen AN, della Malva A, Marino R and Huff-Lonergan E. 2013. Effect of low voltage electrical stimulation on protein and quality changes in bovine muscles during postmortem aging. Meat Science. 3 (July 2013): 289-296. DOI= http://dx.doi.org/10.1016/j.meatsci.2013.02.013

[5] Tokudome W and Wang G. 2012. Similarity dependency of the change in ERP component N1 accompanying with the object recognition learning. International Journal of Psychophysiology. 83 (January 2012): 102-109. DOI= http://dx.doi.org/10.1016/10.1016/j.ijpsycho.2011.10.012.

[6] Qamir H and Ing-Marie J. 2007. A Multi-modal architecture for intelligent decision making in cars. Springer-Verlag Berlin Heidelberg.

[7] Lange K and Schnuerch R. 2014. Challenging perceptual tasks require more attention: The influence of task difficulty on the N1 effect of temporal orienting. Brain and Cognition. 84 (February 2014): 153-163. DOI= http://dx.doi.org/10.1016/j.bandc.2013.12.001.

[8] Yeo MVM, Li X, Shen K and Wilder-Smith EPV. 2009. Can SVM be used for automatic EEG detection of drowsiness during car driving? Safety Science. 47 (January 2009): 115124. DOI= http://dx.doi.org/10.1016/j.ssci.2008.01.007.

[9] Herring S and Hallbeck MS. 2007. The effects of distance and height on maximal isometric push and pull strength with reference to manual transmission truck drivers. International Journal of Industrial Ergonomics. 37 (August 2007): 685696. DOI= http://dx.doi.org/10.1016/j.ergon.2007.05.003. 